\title{
Frankreich und die Türkei - Machtpoker ohne Sieger
}

\author{
Ronja Kempin*
}

In the summer of 2020, Franco-Turkish relations deteriorated dramatically. Since then, Paris has accused Ankara of deliberately influencing the Turkish diaspora in France in order to undermine the values of the French Republic. In terms of foreign policy, France criticizes Turkey for encircling the European Union (EU) and its member states with the consequence of deliberately acting against their interests. Neither the ideological nor the geopolitical power poker of the two states has produced a winner. The Franco-Turkish rivalries offer the EU the opportunity to undertake a comprehensive reorientation of its Turkey policy. The expansion of its conditionality to include domestic political issues is just as important as an improved division of roles and work in its institutions and the geopolitical view of the respective neighbourhood.

Frankreich und die Türkei haben sich im vergangenen Jahr immer weiter voneinander entfernt. Über viele Wochen hinweg bestimmten die ungewöhnlich scharfen Anschuldigungen und Beleidigungen, die sich die Präsidenten Frankreichs, Emmanuel Macron, und der Türkei, Recep Tayyip Erdoğan, wechselseitig zuteilwerden ließen, auch die Beziehungen der Europäischen Union (EU) zur Türkei. Im Herbst 2020 forderte Präsident Macron seine Partner in der EU dazu auf, „mit der Regierung von Präsident Erdoğan, die sich heute in einer inakzeptablen Weise verhält, klar und entschieden um[zu]gehen “. ${ }^{\text {Die }}$ Unterzeichnung „inakzeptabler Vereinbarungen mit der libyschen Einheitsregierung, in denen die legitimen Rechte Griechenlands negiert werden“ und die „inakzeptablen“ türkischen Bohrungen vor der Küste Zyperns führten den französischen Präsidenten Macron zu der Feststellung, „dass die Türkei kein Partner in dieser Region mehr ist“. ${ }^{2}$ Im Mai 2021 schlussfolgerten die Mitglieder des Europäischen Parlaments in einem Bericht, „dass [...] einseitige Maßnahmen im östlichen Mittelmeerraum sowie deutliche und bisweilen provokative, gegen die EU und ihre Mitgliedstaaten gerichtete Aussagen die Beziehungen zwischen der EU und der Türkei auf einen historischen Tiefpunkt gebracht haben $[\ldots]^{\text {“. }}$ Sowohl der Zustand dieser Beziehung als auch ihr Rahmen müssten gründlich überdacht werden. ${ }^{3}$

Frankreichs Türkei-Politik ist in der EU gleichwohl nicht mehrheitsfähig. Vielmehr stehen ihr einige Mitgliedstaaten, darunter auch die Bundesrepublik Deutschland, kritisch

* Dr. Ronja Kempin, Senior Fellow, Forschungsgruppe EU/Europa, Deutsches Institut für Internationale Politik und Sicherheit, Stiftung Wissenschaft und Politik, Berlin.

Dieser Beitrag basiert auf einer früheren Diskussion der Thematik in Ronja Kempin: Frankreich und die Türkei - Entfremdung und strategische Rivalität, in: Ronja Kempin (Hrsg.): Frankreichs Außen- und Sicherheitspolitik unter Präsident Macron. Konsequenzen für die deutsch-französische Zusammenarbeit, SWP-Studie 4, März 2021, S. 44-47, abrufbar unter: https://www.swp-berlin.org/publications/products/studien/2021S04_Macron.pd f (letzter Zugriff: 15.6.2021).

1 Reuters: La Turquie n’est plus un partenaire en Méditerranée orientale, dit Macron, 10. September 2020 Übersetzung der Autorin.

2 Ebenda.

3 Europäisches Parlament: Berichte 2019-2020 über die Türkei. Entschließung des Europäischen Parlaments vom 19. Mai 2021 zu den Berichten 2019-2020 der Kommission über die Türkei (2019/2176(INI)), Wahlperiode 2019-2024, angenommener Text, 19. Mai 2021, P9_TA(2021)0243, hier Punkt 1. 
gegenüber. Hierzulande wird die „destabilisierende Politik“ der Türkei in Libyen und im östlichen Mittelmeerraum zwar ebenfalls nicht länger akzeptiert. Für Berlin kann indes allein der Dialog die Spannungen mit der Türkei auflösen. ${ }^{4}$ Während Deutschland seinen außenpolitischen Leitlinien „Gesprächsbereitschaft“ und „Entspannungspolitik“ treu bleibt und sich etwa beim Gasstreit im östlichen Mittelmeer nicht eindeutig an die Seite Griechenlands stellte, fordert Frankreich seine Partner in der EU immer wieder dazu auf, Sanktionen gegen Ankara zu verhängen, um der Aggressivität der Türkei Einhalt zu gebieten.

Wie ist die harte Haltung Frankreichs gegenüber der Türkei zu erklären? Welche Einflussfaktoren bestimmen den Kurs des französischen Präsidenten und welche Ziele verfolgt er in Bezug auf die Türkei? Diese Fragen sollen in diesem Artikel durch eine intensive Analyse der Themen beantwortet werden, die in den zurückliegenden Monaten für besondere Irritationen zwischen Frankreich und der Türkei geführt haben. Dazu wird der Blick zunächst auf die Verhandlungen um eine EU-Vollmitgliedschaft der Türkei gerichtet, um im Anschluss kurz auf die verbalen Auseinandersetzungen zwischen Frankreichs Präsidenten Macron und dem türkischen Präsidenten Erdoğan näher einzugehen, deren Schärfe für Verbündete unüblich ist. Die „innenpolitischen“ Themen der Religionsfreiheit und der Beeinflussung der türkischen Diaspora werden als zentrale Konfliktfelder in der Beziehung beider Länder identifiziert. Anschließend werden die bedeutsamen außenpolitischen Konfliktfelder östliches Mittelmeer und Afrika eingehender untersucht. Insgesamt werden drei gewichtige Schwächen europäischer Politik offengelegt: Obgleich die Grenzen zwischen Innen- und Außenpolitik immer deutlicher verschwimmen, gelingt es Brüssel und den Mitgliedstaaten erstens kaum, umfassende Antworten etwa darauf zu finden, wie sowohl einer Einflussnahme auf Diaspora-Gruppen Einhalt geboten als auch die geopolitische Verbrüderung mit autoritären und antiwestlichen Kräften gestoppt werden kann. Zum Zweiten tun sich die Mitgliedstaaten (bekanntermaßen) schwer damit, eine außenpolitische Gesamtschau vorzunehmen und daraus ein geeignetes Paket an Gegenmaßnahmen abzuleiten. Drittens offenbart die innenpolitische Dimension der französisch-türkischen Auseinandersetzung, dass es zwischen den Mitgliedstaaten an der Bereitschaft wie den Möglichkeiten mangelt, „best practices“ im Umgang mit externer Einflussnahme auszutauschen. Die Konsequenzen für die EU-Politik gegenüber der Türkei sowie für die EU-Integration werden im letzten Abschnitt erörtert.

\section{Eine bilaterale Beziehung in der Abwärtsspirale}

Frankreich ist nicht unschuldig daran, dass sich die EU-Türkei-Beziehungen in den vergangenen 15 Jahren beständig verschlechtert haben. Unter Staatspräsident Nicolas Sarkozy waren es die Mitgliedstaaten, die Fortschritte auf dem Weg zu einer EU-Vollmitgliedschaft der Türkei blockierten. Gemeinsam mit der Republik Zypern stimmte Paris dagegen, dass im Beitrittsprozess weitere Verhandlungskapitel geöffnet werden.

4 Rede des Außenministers Heiko Maas anlässlich der Konferenz der Botschafterinnen und Botschafter der Französischen Republik, Paris, 31. August 2020, abrufbar unter: https://www.auswaertiges-amt.de/de/newsroom/ma as-boko-paris/2379972 (letzter Zugriff: 15.6.2021).

integration - 3/2021 


\section{Frankreichs frühes Veto gegen die EU-Mitgliedschaft der Türkei}

Bereits im November 2002 hatte der Vorsitzende des europäischen Verfassungskonvents und ehemalige französische Staatspräsident Valérie Giscard d'Estaing eine Mitgliedschaft der Türkei in der EU öffentlich ausgeschlossen. Vor Journalisten bekundete er: „Ich sage meine Meinung: es ist das Ende der Europäischen Union! [...] Die Türkei ist ein Land, das Europa nahesteht, ein wichtiges Land, das eine echte Elite hat, aber es ist kein europäisches Land. [...] Seine Hauptstadt liegt nicht in Europa, es hat 95 Prozent seiner Bevölkerung außerhalb Europas, es ist kein europäisches Land. " ${ }^{5}$ Im Jahre 2004 schloss sich Sarkozy, damals Vorsitzender der konservativen Partei „Union pour un mouvement populaire“ (UMP), diesem Standpunkt an. In einem Fernsehinterview erklärte er: „Wenn die Türkei europäisch wäre, wüssten wir das. "6 Als Staatspräsident plädierte Sarkozy ab 2007 für eine „privilegierte Partnerschaft“ zwischen der Türkei und der EU. Auch Präsident Macron sieht die Türkei nicht als EU-Mitglied. Im August 2018 stellte er vor den Botschafterinnen und Botschaftern seines Landes klar: „Denken wir, dass wir weiter über den Beitritt der Türkei zur Europäischen Union verhandeln können, wenn das täglich vom türkischen Präsidenten bekräftigte Projekt [...] ein panislamisches Projekt ist, das regelmäßig als antieuropäisch dargestellt wird und dessen regelmäßige Maßnahmen unseren Prinzipien eher zuwiderlaufen? Definitiv nicht. “7 Diese Spannungen haben dazu geführt, dass französische Unternehmen oftmals von Ausschreibungen in der Türkei ausgeschlossen wurden. Aus diesem Grund ist Frankreich in der Türkei wirtschaftlich ins Hintertreffen geraten. Einer Modernisierung der Zollunion steht das Land entsprechend kritisch gegenüber. ${ }^{8}$

\section{Verbale Attacken und Entgleisungen}

Der Sommer 2020 führte zu einer signifikanten Verschlechterung der bilateralen Beziehungen zwischen Frankreich und der Türkei und markierte den Beginn einer regelrechten Abwärtsspirale. Einen Vorgeschmack auf die Krise in den Beziehungen lieferten die beiden Präsidenten bereits im November 2019. In einem Interview mit der britischen Wochenzeitung „The Economist“ hatte Präsident Macron der NATO u.a. wegen der international kritisierten Offensive der Türkei gegen kurdische Milizen in Nordsyrien den „Hirntod“ attestiert. ${ }^{9}$ Ankara warf er wegen der Offensive ein „unkoordiniertes, aggressives“ Vorgehen vor, das die Sicherheitsinteressen aller NATO-Staaten berühre. Präsident Erdoğan konterte, „solche Äußerungen passen nur zu Leuten deiner Art, die im Zustand des Hirntods sind““ ${ }^{10}$ Ende Juni 2020 wählte Macron pikanterweise den deutsch-französischen Gipfel auf Schloss

5 Vgl. dazu Le Monde: Pour ou contre l'adhésion de la Turquie à l'Union européenne, 8. November 2002. Übersetzung der Autorin.

6 Wörtlich hatte Nicolas Sarkozy gesagt: „Si la Turquie était européenne, cela se saurait.“ Vgl. dazu L'Obs: Turquie: Sarkozy contredit Chirac, 20. Dezember 2004. Übersetzung der Autorin.

7 Rede des französischen Präsidenten auf der Konferenz der Botschafter, Paris, 27. August 2018, abrufbar unter: https://www.elysee.fr/emmanuel-macron/2018/08/27/discours-du-president-de-la-republique-a-la-conference -des-ambassadeurs (letzter Zugriff: 15.6.2021). Übersetzung der Autorin.

8 Aurélien Denizeau/Dorothée Schmid: France and the Modernization of the EU-Turkey Customs Union. Interests and Obstacles, in: Institut français des relations internationales, Notes de l'Ifri, Juli 2020, abrufbar unter: https://www.ifri.org/sites/default/files/atoms/files/denizeau_schmid_eu-turkey-customs-union_2020.p df (letzter Zugriff: 15.6.2021).

9 The Economist: Emmanuel Macron warns Europe: NATO is becoming brain-dead, 7. November 2019.

10 Vgl. dazu Der Spiegel (online): Erdogan attestiert Macron „Hirntod“ - Paris bestellt türkischen Botschafter ein, 29. November 2019. 
Meseberg, um der Türkei eine „historische und kriminelle Verantwortung“ im libyschen Bürgerkrieg zu attestieren. ${ }^{11}$ Die Türkei gebe vor, Mitglied der NATO zu sein und als solches geachtet zu werden. Sie halte sich jedoch an keine der auf der Berliner Libyen-Konferenz getroffenen Vereinbarungen. Mehr noch: Ankara habe seine militärische Präsenz im Land ausgeweitet und massenhaft djihadistische Söldner aus Syrien nach Libyen gebracht. ${ }^{12}$ Präsident Erdoğan wehrte diese Vorwürfe ab und gab Macron zu verstehen, dass „niemand sich im Spiegel für einen Riesen halten sollte“ ${ }^{13}$ Im Oktober 2020 verlagerte sich die Auseinandersetzung der beiden Präsidenten auf innenpolitische Themen. Am Nachmittag des 16. Oktober 2020 wurde der Lehrer Samuel Paty auf offener Straße von einem islamistisch motivierten 18-Jährigen mit russisch-tschetschenischen Wurzeln enthauptet, nachdem er im Unterricht Karikaturen des Propheten Mohammed gezeigt hatte. Präsident Macron kündigte daraufhin dem radikalen Islam in Frankreich den Kampf an. Moscheen und andere muslimische Einrichtungen sollten stärker kontrolliert und per Gesetz sollte ein mit den Werten der Republik vereinbarer „französischer Islam“ geschaffen werden. Nach den Worten seines Präsidenten werde Frankreich nicht auf Zeichnungen und Karikaturen verzichten, auch wenn sie sich auf den Propheten Mohammed beziehen. Eine Woche nach dem Mord an Paty wetterte der türkische Präsident auf einem Kongress seiner „Adalet ve Kalkınma Partisi” (AKP) gegen „besorgniserregende Anzeichen einer wachsenden Islamfeindlichkeit in Europa“. "14 „Was für ein Problem hat diese Person namens Macron mit dem Islam und Muslimen?", fragte Erdoğan seine Parteianhänger. ${ }^{15}$ Macron verstehe die Glaubensfreiheit nicht. Er gehöre in psychologische Behandlung; während Waren aus Frankreich in der islamischen Welt boykottiert werden sollten. ${ }^{16}$ Im Dezember 2020 erreichte die Krise ihren vorläufigen Höhepunkt. Informationen des Nachrichtenportals Al Jazeera zufolge soll der türkische Präsident Erdoğan Anfang Dezember 2020 angemerkt haben: „Macron ist eine Last für Frankreich. Macron und Frankreich gehen durch einen sehr gefährlichen Zeitabschnitt. Ich hoffe, dass Frankreich Emmanuel Macron und den mit ihm verbundenen Ärger so schnell wie möglich loswird““ ${ }^{17}$

\section{Innenpolitische Streitthemen}

Die Auseinandersetzungen zwischen Paris und Ankara begrenzten sich „innenpolitisch“ in den vergangenen Jahren vornehmlich auf Fragen zur Anerkennung des Völkermordes an den Armeniern durch Frankreich. Unter der Präsidentschaft von Macron ist die türkische Einflussnahme auf die muslimische Diaspora in Frankreich als strukturelle Veränderung handlungsleitend.

11 Le Figaro: Libye: Macron condamne la «responsabilité historique et criminelle» de la Turquie, 29. Juni 2020.

12 Ebenda.

13 Vgl. dazu Iyad Dakka: Will Either Macron or Erdogan Back Down in the Eastern Mediterranean?, in: World Politics Review (online), 9. September 2020. Übersetzung der Autorin.

14 Schweizer Radio und Fernsehen News International: Erdogan beleidigt Macron: Frankreich ruft Botschafter zurück, 25. Oktober 2020 .

15 Ebenda.

16 Ebenda.

17 Marvin Ziegele: Türkei - Erdogan stichelt gegen Macron: „Hoffe, dass Frankreich ihn so schnell wie möglich loswird“, in: Frankfurter Rundschau (online), 4. Dezember 2020. 


\section{Völkermord an den Armeniern}

Das „älteste“ Streitthema zwischen Paris und Ankara ist die Anerkennung des Völkermordes an den Armeniern. Am 18. Januar 2001 hatte die französische Nationalversammlung einen Gesetzentwurf verabschiedet, demgemäß „Frankreich den armenischen Völkermord von 1915 öffentlich anerkennt" ${ }^{18}$ Etwa 500.000 bis 600.000 Armenier leben heute in Frankreich, wobei es sich um die drittgrößte armenische Diaspora der Welt handelt. Weil Frankreich bis in die 1920er Jahre Kilikien und Teile Süd-Anatoliens besetzt hatte, flohen viele Armenier am Ende des Ersten Weltkrieges insbesondere in die französischen Hafenstädte Marseille und Valence. Bereits 1972 hatte Frankreich als erstes Land in Europa eine Gedenkstätte für den Völkermord an den Armeniern eingeweiht (in Décines-Charpieu, einer Kleinstadt im Osten von Lyon).

In den Jahren 2012 und 2017 wollte die französische Nationalversammlung sogar noch einen Schritt weiter gehen. Mit Unterstützung der damaligen Staatspräsidenten Nicolas Sarkozy und François Hollande verabschiedete sie Gesetze, wonach eine Leugnung des Völkermordes strafrechtliche Konsequenzen gehabt hätte. Beide Gesetzesinitiativen wurden jedoch vom französischen Verfassungsgericht unter Verweis auf die Meinungsfreiheit und den Umstand, dass der Völkermord von 1915 international nicht anerkannt sei, gekippt. ${ }^{19}$ Der langsamen Verschlechterung der bilateralen Beziehungen mit der Türkei konnte diese Entscheidung nicht entgegenwirken. Macron löste im Januar 2019 ein Wahlversprechen ein und erklärte den 24. April zum „nationalen Gedenktag für den Völkermord an den Armeniern“ (journée nationale de commémoration du génocide arménien). ${ }^{20}$ Mit dieser Geste schaue Frankreich sinnbildlich „der Geschichte ins Gesicht“. Die Türkei reagierte prompt und ungehalten: sie verurteile jeden Versuch Macrons, „historische Ereignisse zu einer politischen Frage“ zu machen. ${ }^{21}$

\section{Beeinflussung der Diaspora}

Die Türkei wendet sich darüber hinaus gegen das „Gesetz zur Anerkennung des Völkermords an den Armeniern“ - und dies verstärkt durch Aktivitäten in Frankreich. In den vergangenen Jahren hat Ankara sein Netzwerk an Institutionen, Vereinigungen und Bewegungen wie auch Medien in Frankreich massiv ausgeweitet. Das traditionell starke Netzwerk der türkischen säkularen Vereinigungen ist in Frankreich mittlerweile nahezu vollständig von Organisationen verdrängt worden, die die nationalistische und religiöse Agenda der AKP fördern. Dieses neue Netzwerk hat den Auftrag, alles anzugreifen, was mit dem „Gesetz zur Anerkennung des Völkermords an den Armeniern“ oder dem „Gesetz zum Verbot sichtbarer religiöser Zeichen in Schulen“ zu tun hat.

Im Februar 2020 machte Präsident Macron darauf aufmerksam, dass sich die Türkei weigere, mit Frankreich sowohl den Türkischunterricht als auch die Entsendung von

18 Assemblée Nationale, Onzième Législature, Session Ordinaire de 2000-2001: Proposition de Loi relative à la reconnaissance du génocide arménien de 1915, 18. Januar 2001, abrufbar unter: http://www.assemblee-nationa le.fr/11/ta/ta0611.asp (letzter Zugriff: 15.6.2021). Übersetzung der Autorin.

19 Vgl. dazu François Tonneau: Le Conseil constitutionnel défait la loi sur le génocide arménien, in: La Provence, 21. Januar 2017.

20 Vgl. dazu Le Figaro: Le 24 avril deviendra journée nationale de commémoration du génocide arménien, 5. Februar 2019.

21 Vgl. dazu Der Spiegel (online): Türkei verärgert über französische Gedenktagspläne, 6. Februar 2019. 
Imamen neu zu verhandeln. Um Schülerinnen und Schüler in ihrer Herkunftssprache und -kultur zu unterrichten, hatte Frankreich bereits im Jahr 1977 mit neun Staaten bilaterale Abkommen abgeschlossen, die es diesen Staaten erlauben, ausgebildete Lehrerinnen und Lehrer zu Unterrichtszwecken nach Frankreich zu entsenden. Rund 80.000 Schülerinnen und Schüler nehmen diese „enseignements en langues et cultures d'origine“ (ELCO) heute wahr; ungefähr 14.000 von ihnen die Angebote in türkischer Sprache. Die staatliche Schulaufsicht hatte bislang allerdings keine Kontrolle über die Inhalte der Lehrangebote. Es mehrten sich die Hinweise, dass die entsandten Lehrkräfte zumeist Inhalte vermittelten, welche den Grundgedanken der Französischen Republik zuwiderlaufen. Aus diesem Grund stellt Frankreich dieses System nunmehr um. Mit acht der neun Staaten wurde vereinbart, dass sowohl das Lehrangebot als auch die Lehrbefähigung der staatlichen Kontrolle Frankreichs unterliegen sollen. Weil Ankara diesen Schritt ablehnt, hat Paris das ELCO-System im September 2020 eingestellt. ${ }^{22}$

Die Differenzen zwischen Paris und Ankara konnten schließlich auch mit Blick auf die Entsendung von Imamen nicht ausgeräumt werden. Im Februar 2020 bekannte Präsident Macron, wie wichtig für Frankreich die Gewissheit ist, dass alle Personen, die im Namen einer Religion tätig sind, auch die Werte der Republik respektieren. ${ }^{23}$ Deshalb sei es erforderlich, dass sich Frankreich ab 2020 schrittweise von seinem althergebrachten, oftmals als „konsularischer Islam“ bezeichneten System trennen muss, indem nicht mehr jährlich sogenannte abgeordnete Imame und von anderen Regierungen ernannte und ausgebildete Sänger von Psalmodien in das Land immigrieren dürfen. ${ }^{24}$ Der Türkei war es bisher gelungen, die Hälfte der 300 nach Frankreich abgeordneten Imame zu stellen. Im Jahr 2017 hatte der französische Rat der Muslime erstmals seit seiner Gründung im Jahr 2003 einen Vertreter des türkischen Islams für zwei Jahre an seine Spitze gewählt. ${ }^{25}$

Die Türkei hatte ihrerseits im November 2019 vier türkische Staatsbürger verhaftet, welche sie der "politischen und militärischen“ Spionage im Namen Frankreichs beschuldigte. Wie sich herausstellte, arbeiteten die verhafteten Personen an einer Untersuchung von Aktivitäten der türkischen Führung in Frankreich in Bezug auf religiöse Angelegenheiten (Diyanet in der Türkei und Ditib im Ausland) wie auch zu ihrer Strategie der religiösen Bekehrung, die sie in französischen Vorstädten und in sozialen Netzwerken verfolgt. Pikanterweise hatte die türkische Tageszeitung Sabah, das wichtigste Sprachrohr der islamischkonservativen Regierung, die Namen der Spione veröffentlicht, die dem französischen Auslandsgeheimdienst „Direction Générale de la Sécurité Extérieure“ (DGSE) angehörten.

22 Wörtlich sagte Präsident Macron: „Si aucune solution n'est trouvée avec la Turquie et qu'elle refuse ce dispositif, nous mettrons fin à toute enseignement obligatoire, et donc nous construirons, avec l'ensemble de nos concitoyens, des associations concernées, des alternatives pour bâtir une offre périscolaire renouvelée, trouver des enseignants en langue et culture turque, Française et Français, maîtrisant notre langue, et nous assurerons précisément ces enseignements nous-mêmes parce qu'il est important que toutes les Françaises et tous les Français d'origine turque puissent continuer aussi d'avoir ces enseignements, de continuer à maîtriser une langue étrangère qui est parfois celle de leur famille, de la même manière quand ils sont binationaux, ce qui arrive aussi pour une partie d'entre eux, mais qu'ils le fassent dans un cadre qui soit pleinement républicain." Siehe Élysée: Transcription du propos liminaire du Président de la République lors du point-presse à Mulhouse, 18. Februar 2020, abrufbar unter: https://www.elysee.fr/front/pdf/elysee-module-15216-fr.pdf (letzter Zugriff: 15.6.2021).

23 Ebenda.

24 Ebenda.

25 Piotr Smolar: La France contre la Turquie, aux racines de l'affrontement, in: Le Monde, 10. Juli 2020.

integration - 3/2021 
In der Regel werden die Behörden des betreffenden Landes vor der Veröffentlichung solcher Angelegenheiten informiert, damit sie notwendige Vorkehrungen zur Sicherheit ihrer Agenten treffen können. ${ }^{26}$

\section{Das Gesetz zur Stärkung republikanischer Prinzipien}

Bereits während seines Präsidentschaftswahlkampfes im Jahr 2017 hatte der französische Präsident Macron angekündigt, eine Grundsatzrede zum Islam zu halten. Letztlich vergingen dreieinhalb Jahre - in Frankreich hatten immer wieder Terrorangriffe stattgefunden und der Präsident musste sich deshalb den Vorwurf gefallen lassen, zu lange untätig gewesen zu sein - bis er sich Anfang Oktober 2020 in seiner Rede in Les Mureaux zu einer „Republik der Taten“ bekannte:

„Das Problem ist der islamistische Separatismus. Dieses bewusste, theoretisch untermauerte, politisch-religiöse Vorhaben, das durch wiederholte Diskrepanzen zu den Werten der Republik Gestalt annimmt, oft zur Herausbildung einer Gegengesellschaft führt und sich in Schulabbrüchen von Kindern und der Entwicklung sportlicher, kultureller und kommunitarisierter Praktiken äußert, die als Vorwand für das Lehren von Prinzipien dienen, welche nicht den Gesetzen der Republik entsprechen. Das ist Indoktrinierung und, mittels dieser, eine Negierung unserer Prinzipien wie der Gleichstellung von Frau und Mann und der Menschenwürde. Das Problem ist diese Ideologie, die behauptet, dass ihre eigenen Gesetze über denen der Republik stehen. Ich verlange von keinem unserer Bürger, zu glauben oder nicht zu glauben, ein wenig oder moderat zu glauben - das ist nicht Sache der Republik. Doch ich verlange von jedem Bürger, egal welcher Religion er angehört oder nicht, ausnahmslos alle Gesetze der Republik zu achten. “27

Seither diskutiert Frankreich über das „Gesetz zur Stärkung des Respekts vor den Prinzipien und Werten der Republik“ “28 Das Gesetzesvorhaben, das zunächst „Gesetz gegen Separatismus" hieß, wurde nach der Ermordung von Samuel Paty und dem Anschlag in der Basilika Notre-Dame-de-l'Assomption in Nizza am 29. Oktober 2020, als es drei Tote gab, verschärft. In Frankreich entfachte der Gesetzentwurf insbesondere Diskussionen zu der Fragestellung, inwiefern Muslime pauschal verurteilt und stigmatisiert würden und ob nicht noch weit härtere Maßnahmen ergriffen werden müssten, um Terroranschlägen und Angriffen auf die Werte Frankreichs entgegenzuwirken. Die Regierung der Türkei reagierte hingegen empört auf die schärfere Kontrolle von Moscheen und Islamverbänden. Im Oktober 2020 standen nach Angaben des französischen Innenministers Gérald Darmanin 76 Moscheen unter dem Verdacht eines radikalen Separatismus. Über Twitter erklärte der Innenminister: „Dort predigen Imame, die im Fokus der Geheimdienste stehen. Sie versto-

26 Ebenda.

27 Zitiert nach Frankreich in Deutschland, Französische Botschaft in Berlin: Auszüge aus der Rede des französischen Staatspräsidenten zum Thema Kampf gegen Separatismus, 2. Oktober 2020, abrufbar unter: https://de.a mbafrance.org/Auszuge-aus-der-Rede-des-franzosischen-Staatsprasidenten-zum-Thema-Kampf-gegen (letzter Zugriff: 15.6.2021).

28 Assemblée Nationale, Quinzième Législature: Projet de loi no 3649 confortant le respect des principes de la République, 9. Dezember 2020, abrufbar unter: https://www.assemblee-nationale.fr/dyn/15/textes/115b3649_p rojet-loi\#tocUniqueId5 (Zugriffsdatum: 15.6.2021). 
ßen gegen unsere Werte, erkennen die Gleichstellung von Mann und Frau nicht an, sind antisemitisch, predigen Hass auf Frankreich. "29 Gemäß dem Gesetzesvorhaben sollen Imame zukünftig in Frankreich ausgebildet und die ausländische Finanzierung von islamischen Organisationen soll stärker überwacht werden. Die neun wichtigsten Vereinigungen der Muslime in Frankreich wurden im November 2020 von Präsident Macron dazu aufgefordert, bis zum 18. März 2021 eine Charta vorzulegen, die über ihr Religionsverständnis und insbesondere zu ihrem Verhältnis zum republikanischen Wertekanon Auskunft erteilt. ${ }^{30}$

\section{Geopolitische Konfrontationen}

$\mathrm{Zu}$ diesen Auseinandersetzungen sind geopolitischen Rivalitäten hinzugekommen. Paris bewertet die Außenpolitik Ankaras im östlichen Mittelmeer, in Afrika und jüngst auch im Kaukasus als „antieuropäisch“. Es sieht aber auch seine Sonderstellung im Mittelmeer sowie auf dem afrikanischen Kontinent in Gefahr. Im östlichen Mittelmeerraum verstärkte Frankreich im Sommer 2020 seine Luft- und Seestreitkräfte. Mit zwei zusätzlichen Rafale-Kampfflugzeugen und einem Marineschiff reagierte Paris auf die Wiederaufnahme der umstrittenen Gasförderung durch die Türkei südlich der Republik Zypern sowie auf die Suche nach neuen Erdgasreserven entlang der griechischen Küste. Frankreich führte mit Griechenland und Zypern gemeinsame Militärmanöver durch und unterstützte beide EU-Mitgliedstaaten in ihrer Forderung, den türkischen Präsidenten mit Sanktionen zu belegen und „rote Linien“ zu formulieren. Frankreichs Parteinahme im jahrzehntealten griechisch-türkischen Konflikt gründet vornehmlich in der Angst vor einer neuen Ordnung im östlichen Mittelmeer sowie in (rüstungs-)wirtschaftlichen Aspekten.

\section{Angst vor einer neuen Ordnung im östlichen Mittelmeer}

Im Sommer 2020 unterstrich Präsident Macron bei einem Besuch von Bundeskanzlerin Angela Merkel einmal mehr, dass sich Frankreich als Mittelmeermacht verstehe. Mehr noch: Im Mittelmeerraum reklamiert es eine Vorreiterrolle für sich. Die Regierung in Paris begründet diese Rolle mit dem Umstand, dass Frankreich die einzige Mittelmeermacht mit einem ständigen Sitz im Sicherheitsrat der Vereinten Nationen (UN) sei und über die schlagkräftigsten Streitkräfte aller Anrainerstaaten verfüge. Schließlich besitzt Frankreich in der Region sogenannte „soft power“. Diese speist sich aus historischen Verbindungen (so schwierig und komplex diese auch sein mögen) zu den politischen Eliten der Mittelmeeranrainerstaaten sowie einem umfangreichen Netzwerk diplomatischer, kultureller und schulischer Vertretungen. In vielen Ländern des Mittelmeeres wird Französisch gesprochen.

Unter sicherheits- und verteidigungspolitischen Gesichtspunkten ist das östliche Mittelmeer ein wichtiges Element der französischen Sicherheitsstrategie. Die dortige Instabilität bietet zahlreichen djihadistischen Aktivitäten Raum, neue Terroranschläge auch gegen Europa vorzubereiten. Den Gruppierungen des Islamischen Staates (IS) stellt sich Frankreich

29 Zitiert nach Sabine Wachs: Die Werte der Republik gegen Islamismus, in: tagesschau.de, 9. Dezember 2020, abrufbar unter: https://www.tagesschau.de/ausland/europa/frankreich-separatismus-gesetz-101.html (letzter Zugriff: 15.6.2021).

30 Rudolf Walther: Das Gesetz anbeten, nicht den Koran, in: der Freitag, Ausgabe 6/2021, 14. Februar 2021. 
seit dem Jahr 2014 im Rahmen der Luftoperation „Chammal“ entgegen. Aus Jordanien heraus greifen französische Kampfflugzeuge Stellungen des IS (Daech) in Syrien und im Irak an. Darüber hinaus stärkt Paris regionale Akteure, die im Kampf gegen den islamischen Terrorismus als effektiv angesehen werden. Paris setzt dazu in Syrien auf die kurdischen „Volksverteidigungseinheiten“ (Yekîneyên Parastina Gel, YPG). Als die Türkei, die die YPG als Terrororganisation einstuft, im Oktober 2019 eine militärische Offensive gegen die YPG in Nordsyrien startete und der Organisation schwere Verluste zufügte, beurteilte Paris dies als „ernsthaften Angriff auf [seine] Sicherheitsinteressen “. ${ }^{31}$

Gleichzeitig betrachtet Frankreich das östliche Mittelmeer als ein Sprungbrett seiner Machtprojektion in den Nahen Osten so wie das östliche Afrika, weshalb es seine Präsenz in der Region kontinuierlich ausbaut. Im September 2014 hat Paris eine „temporäre Luftwaffenbasis“ (base aérienne projetée, BAP) in Jordanien eröffnet. Die Basis dient als logistische Plattform für seine militärischen Standorte im Irak, den Vereinigten Arabischen Emiraten (VAE) und Djibouti. Mit der Republik Zypern hatte Paris bereits im April 2017 ein Abkommen geschlossen, welches die militärische Zusammenarbeit zwischen den beiden Ländern stärken soll. ${ }^{32}$ Das Abkommen ist am 1. August 2020 in Kraft getreten. ${ }^{33}$ Hierauf unterzeichneten der französische und der zyprische Verteidigungsminister im Mai 2019 eine Absichtserklärung, wonach französische Marineschiffe den zypriotischen Marinestützpunkt Mari anlaufen und nutzen dürfen. ${ }^{34}$ Seit 2017 hat die französische Marine eine Vielzahl gemeinsamer Trainingsübungen mit ihren griechischen und zypriotischen Kollegen sowie mit anderen regionalen Partnern durchgeführt. Paris reagiert damit auf den Rückzug der USA aus der Region, aber auch auf die türkische Marinedoktrin der „Blauen Heimat“ (Mavi Matan). Diese zielt darauf ab, die maritimen Interessen Ankaras im Mittelmeer, in der Ägäis und im Schwarzen Meer besser zu schützen. In den vergangenen Jahren hat die Türkei massiv in den Ausbau ihrer Seestreitkräfte und Werften investiert. ${ }^{35}$ Paris befürchtet infolgedessen, dass Ankara eine neue Ordnung im östlichen Mittelmeer durchsetzen will, wobei dieser Raum in der Türkei vermehrt als „türkische See“ bezeichnet wird. ${ }^{36}$

\section{Wirtschaftliche Aspekte}

Neben der Frage der regionalen Vormachtstellung treiben wirtschaftliche Aspekte die Regierung in Paris dazu an, sich in der Region zu engagieren. Frankreich ist zum einen an einer Mitgliedschaft im „Gasforum Östliches Mittelmeer“ interessiert. Im Januar 2020 ersuchte Paris um Aufnahme in diese regionale Organisation, der Ägypten, Israel, Griechenland, Zypern, Italien, Jordanien und die Palästinensische Autonomiebehörde angehören. Das Gasforum will den Energiebedarf seiner Mitglieder decken, das Gas der Region aber auch zu wettbewerbsfähigen Preisen in die EU exportieren. Gemeinsam mit der

31 Smolar: La France contre la Turquie, aux racines de l'affrontement, 2020.

32 Accord coopération en matière de défense entre le Gouvernement de la République française et le Gouvernement de la République de Chypre, signé à Paris le 4 avril 2017, Annex zum Dekret Nr. 2020-1038 vom 13. August 2020, in: Journal officiel de la République française, Nr. 0200 vom 15. August 2020, Text 3 von 183.

33 Ebenda.

34 Vgl. dazu George Psyllides: Expansion of base for use of French navy a priority says minister, in: Cyprus Mail (online), 22. Mai 2019.

35 Günter Seufert: Die Türkei auf dem Weg zur Seemacht, in: Le Monde diplomatique, 9. Mai 2019.

36 Vgl. dazu Dakka: Will Either Macron or Erdogan Back Down in the Eastern Mediterranean?, 2020. 
italienischen Firma Eni hat der französische Energiekonzern Total Fördergenehmigungen für Gasvorkommen in zypriotischen, griechischen und libanesischen Küstengewässern erhalten. Paris hofft, dass eine verstärkte Einflussnahme auf die Spannungen in der Region seine Aussichten auf eine Vollmitgliedschaft wie auch Zugriffe auf die Förderung, Vermarktung und den Transport des Gases verbessert, die gegenwärtig verhandelt werden. Der Türkei wurde hingegen eine Mitgliedschaft im „Gasforum Östliches Mittelmeer" verwehrt. Entsprechend lehnt Ankara die Organisation ab und wirft ihr vor, die Türkei von der Zusammenarbeit und den Gewinnen auszuschließen. ${ }^{37}$

Frankreich verfolgt zum anderen rüstungspolitische Interessen in der Region. Paris hatte sehr genau beobachtet, dass die griechischen Streitkräfte seit Ausbruch der Finanzund Schuldenkrise nicht modernisiert worden waren. Bereits im Januar 2020 waren Paris und Athen darin übereingekommen, eine „strategische Sicherheitspartnerschaft“ zu begründen. ${ }^{38}$ Im September 2020 gab der griechische Ministerpräsident Kyriakos Mitsotakis bekannt, dass sein Land in den kommenden vier Jahren gut 10 Milliarden Euro für den Kauf von Rüstungsgütern ausgeben werde. ${ }^{39}$ Die Luftwaffe werde 18 Rafale-Kampfflugzeuge von Frankreich kaufen (sechs neue und zwölf gebrauchte). Zwischen 1974 und 2000 hatte Griechenland bei dem französischen Hersteller Dassault Aviation 95 Kampfflugzeuge vom Typ Mirage erworben, dem Vorgänger der Rafale. Möglicherweise kommt auch die französische Naval Group in Griechenland noch zum Zuge: Athen plant den Kauf von vier neuen Fregatten. Für Dassault Aviation kommt diese Kaufankündigung, die noch vertraglich verankert werden muss, genau zur richtigen Zeit. Das Unternehmen muss pro Jahr mindestens elf Rafale-Kampfflugzeuge verkaufen, um wirtschaftlich zu bleiben. Diese Größenordnung übersteigt den Bedarf der französischen Armee, weshalb Paris dem Unternehmen zugesagt hat, es beim Export der Rafale zu unterstützen. Weil Frankreich in den kommenden beiden Jahren keine Rafale-Flugzeuge kaufen wird, internationale Aufträge aber (bedingt durch die COVID-19-Pandemie) ebenfalls ausgeblieben waren, sah sich die Geschäftsführung von Dassault Aviation im Sommer 2020 dazu genötigt, in Gesprächen mit Verteidigungsministern Florence Parly um staatliche Unterstützung zu bitten. Die griechische Kaufankündigung entbindet die französische Luftwaffe von der Pflicht, Lieferungen antizipieren zu müssen. ${ }^{40}$

\section{Konkurrenz auf dem afrikanischen Kontinent}

Der französisch-türkische Antagonismus im östlichen Mittelmeer darf indes nicht den Blick dafür verstellen, dass sich Paris auch auf dem afrikanischen Kontinent von Ankara

37 Karol Wasilewski/Łukasz Maślanka: The Franco-Turkish Tensions, in: The Polish Institute of International Affairs, Bulletin 147, 10. Juli 2020, abrufbar unter: https://www.pism.pl/publications/The_FrancoTurkish_Tensi ons (letzter Zugriff: 15.6.2021); Dakka: Will Either Macron or Erdogan Back Down in the Eastern Mediterranean?, 2020.

38 Erklärung des französischen Präsidenten Emmanuel Macron zu den Beziehungen zwischen Frankreich und Griechenland sowie europäischen Fragen, Paris, 30. Januar 2020, abrufbar unter: https://www.vie-publique.fr/ discours/273141-emmanuel-macron-30012020-france-grece (Zugriffsdatum: 15.6.2021); vgl. dazu Marina Rafenberg: Face aux tensions persistantes avec la Turquie, la Grèce muscle sa défense militaire, in: Le Monde, 16. September 2020.

39 Vgl. dazu Isabelle Chaperon/Piotr Smolar: La Grèce, premier pays européen à acheter le Rafale, in: Le Monde, 13. September 2020.

40 Ebenda. 
zunehmend in die Defensive gedrängt sieht. In Libyen stützte Paris lange Zeit General Chalifa Haftar und hoffte, dass dessen Sieg über die nationale Einheitsregierung das Land schnell stabilisieren würde. In Paris gilt die international anerkannte nationale Einheitsregierung als von der Muslimbruderschaft beeinflusst. Der Türkei lastet Paris an, die Muslimbruderschaft als außenpolitisches Instrument zu nutzen. Die offene militärische Intervention der Türkei zur Unterstützung der nationalen Einheitsregierung hat im ersten Halbjahr den Verlauf des libyschen Bürgerkriegs umgekehrt. Mit Unterstützung der Türkei ist es der in Tripolis ansässigen Regierung gelungen, die von Frankreich, Ägypten, Russland und den VAE ausgestatteten Kräfte General Haftars aus dem Westen Libyens zu vertreiben. Der Türkei nutzte ihr Engagement. Im Juni 2020 vereinbarte Ankara mit der nationalen Einheitsregierung, einen türkischen Marinestützpunkt in Misrata ausbauen zu dürfen. Auf dem wiedereroberten Luftwaffenstützpunkt al-Watiya, 27 Kilometer von der tunesischen Grenze entfernt, konnte Ankara überdies eine Luftwaffenpräsenz einrichten. Schließlich unterzeichneten die Türkei und Libyen im August 2020 eine Absichtserklärung zur Intensivierung ihrer Handels- und Wirtschaftsbeziehungen. ${ }^{41}$ Darin wird Ankara erlaubt, Bauprojekte, die während der Ära des Diktators Muammar al-Gaddafi ausgehandelt wurden, durchzuführen. Ferner wurden neue türkische Investitionen und die Verstärkung des Handels vereinbart. Im Jahr 2019 war die Türkei nach China bereits der größte Exporteur von Waren nach Libyen. ${ }^{42}$ Dass Paris möglicherweise an einem durch RafaleKampfflugzeuge ausgeführten Luftangriff auf den Luftwaffenstützpunkt al-Watiya beteiligt gewesen war, der den neu stationierten türkischen Luftverteidigungsanlagen erheblichen Schaden zugefügt hatte, zeigt, wie stark sich das Land von der türkischen Einflussnahme bedrängt sieht.

Libyen ist nur ein Bestandteil der türkischen Afrikapolitik: Von 2010 bis 2016 eröffnete Ankara 26 Botschaften in Afrika. ${ }^{43}$ Während die türkischen Aktivitäten in der Großregion Ostafrika insbesondere Frankreichs Partnern Ägypten, den VAE und Saudi-Arabien Anlass zur Sorge geben, gerät Paris in West- und Zentralafrika in Bedrängnis. Im Januar 2020 besuchte der türkische Präsident Algerien, das er als „eines der wichtigsten Tore der Türkei zum Maghreb und zu Afrika" bezeichnete, und schloss eine strategische Partnerschaft mit Rabat. Mit Niger vereinbarte die Türkei im Juli 2020 mehrere Abkommen über eine verbesserte wirtschaftliche und verteidigungspolitische Zusammenarbeit. In der Verteidigungspolitik bildet Ankara künftig nigrische Soldaten aus. Für Frankreich gleichermaßen brisant ist der Einstieg der Türkei in die nigrische Bergbauindustrie. Das Uran, welches Frankreich für den Betrieb seiner Kernkraftwerke benötigt, stammt zu einem Drittel aus Niger. ${ }^{44}$

Die EU nimmt auf dem afrikanischen Kontinent weiter die Rolle des Zaungastes ein. Brüssel will die wirtschaftliche und demokratische Entwicklung des Kontinents fördern. Jedoch betrachtet es seine südliche Nachbarschaft nicht als geopolitischen Raum. Dies dürfte Frankreich dazu veranlassen, künftig noch enger mit Ägypten und den VAE zu-

41 Libyan Express: Libya, Turkey sign MoU in trade and economy cooperation, 13. August 2020.

42 Michaël Tanchum: Turkey Advances in Africa against Franco-Emirati-Egyptian Entente, in: The Turkey Analyst, 25. August 2020.

43 Ebenda.

44 Ebenda. 
sammenzuarbeiten, um einer Ausweitung des türkischen Einflusses im Maghreb und in Subsahara-Afrika Einhalt zu gebieten.

\section{Französisch-türkische Rivalität: Eine Chance für die EU}

Die französisch-türkische Rivalität ist vielschichtig und speist sich aus unterschiedlichen Faktoren. Wenngleich die Töne zwischen Paris und Ankara jüngst wieder versöhnlich klangen, sieht der französische Präsident Ankaras Versuche, Einfluss auf die Innenpolitik von EU-Mitgliedstaaten zu nehmen, ebenso kritisch wie das Streben der Türkei, Frankreich und die EU geopolitisch einzuhegen. Im Jahr 2020 hat insbesondere das Ringen um die Vorherrschaft im östlichen Mittelmeer sowie auf dem afrikanischen Kontinent auf die NATO und in die EU ausgestrahlt und das Binnenverhältnis beider Organisationen empfindlich gestört. Weder in der EU noch in der NATO konnte Macron seine Partner von einer härteren Gangart gegenüber der Türkei überzeugen. Im Juni 2020 etwa folgten lediglich acht der 30 NATO-Mitglieder dem Antrag Frankreichs, die Türkei für einen unter Alliierten unzulässigen Vorgang zu verurteilen: Ein türkisches Kriegsschiff nahm im Juni 2020 mehrfach die französische Fregatte „Courbet“ ins Visier, die im Rahmen der NATO-Operation „Sea Guardian“ das UN-Waffenembargo vor der Küste Libyens kontrollierte. Umgekehrt scheiterte die EU wiederholt mit ihrer Politik einer positiven Türkei-Agenda. Im Herbst 2020 zog Ankara sein Forschungsschiff „Oruç Reis“ kurz vor der Sitzung des Europäischen Rates aus Gewässern zurück, die Griechenland zu seiner Exklusiven Wirtschaftszone (EWZ) rechnet. Zwei Tage nach der Sitzung des Rates wurde die „Oruç Reis“ jedoch wieder in die umstrittenen Gewässer zurückbeordert.

So bietet die französisch-türkische Rivalität der EU die Gelegenheit, die angestrebte Neuausrichtung ihrer Türkeipolitik umfassend zu gestalten. Ein Dreiklang bietet sich dabei an: Zum einen sollten innenpolitische Themen von der EU in ihre Politik der Konditionalität aufgenommen werden. Die analysierten Dispute zwischen Frankreich und der Türkei sind kein Einzelfall. Auch in anderen Mitgliedstaaten versucht Ankara, die eigene Diaspora zu beeinflussen. Dass sich die Türkei in religiöse, kulturelle oder schulische Belange in EU-Mitgliedstaaten einmischt, sollte künftig unterbunden werden und mit sanktionierenden Maßnahmen einhergehen. Zweitens sollte die EU die Debatten um eine Neugestaltung ihrer Türkeipolitik nutzen, das Zusammenspiel ihrer Organe zu verbessern. Der Fokus des Europäischen Parlaments und der Europäischen Kommission liegt weiterhin auf dem Beitrittsprozess und somit auf Demokratie und Rechtsstaatlichkeit. Der Europäische Rat nimmt indes zunehmend die Geopolitik in den Blick. Die Verzahnung beider Ansätze fehlt bislang. Die EU wird geschwächt, weil sich ihre Institutionen wechselseitig mit Vorhaltungen und Diffamierungen auseinandersetzen müssen. Schließlich bietet die französisch-türkische Rivalität der EU die Chance, ihr außenpolitisches Profil zu stärken. Es steht außer Frage, dass sich die EU-Mitgliedstaaten im Europäischen Rat um einen Ausgleich zwischen Ankara und Paris bemühen müssen. Grundlage ihres Handelns sollte die Übereinkunft sein, dass der Türkei die Vollendung der Zollunion erst dann angeboten wird, wenn sich Ankara bereit erklärt, seine gegen EU-Interessen gerichtete Politik in den Nachbarländern einzustellen. Paris benötigt mehr Unterstützung von seinen EU-Partnern, die begreifen müssen, dass ihre Nachbarschaft wie auch sie selbst zu einem geopolitischen Spielfeld geworden sind, auf dem jede Enthaltung bedeutet, ausgebootet zu werden.

integration $-3 / 2021$ 